\title{
New oxygen-iodine hybrid laser technology
}

\section{David Carroll and Wayne Solomon}

A high-power laser has been developed that combines the beam quality of gas lasers with the advantages of electrical pumping.

The high-power laser community has long desired a hybrid laser system that combines the advantages of electrically driven lasers and the high beam quality of gas lasers. For more than 30 years, an electrically driven oxygen-iodine laser has been widely considered a likely candidate system. However, it was not until 2004 that we overcame the technological difficulties and successfully demonstrated gain and lasing. ${ }^{1,2}$ This was possible thanks to significant knowledge-base improvements by colleagues at Emory University, Physical Sciences Inc., Iowa State University, and the Air Force Institute of Technology. Together, we had the the combined knowledge of fluid dynamics, kinetics, electrodynamics, and laser physics needed to successfully tackle this highly complex, multidisciplinary problem.

The hybrid electric oxygen-iodine laser (ElectricOIL) system, shown schematically in Figure 1, uses an electric discharge to pump molecular oxygen to its first electronic excited state, $\mathrm{O}_{2}\left(a^{1} \Delta\right)$, also denoted $\mathrm{O}_{2}(\mathrm{a})$. Helium is used as a buffer gas to lower the discharge gas temperature. The $\mathrm{O}_{2}(\mathrm{a})$ state then transfers its energy to atomic iodine, and laser oscillation is obtained from the $\mathrm{I}\left({ }^{2} \mathrm{P}_{1 / 2}\right) \rightarrow \mathrm{I}\left({ }^{2} \mathrm{P}_{3 / 2}\right)$ transition at $1315 \mathrm{~nm}$. In the current design, atomic iodine is created through reactions between molecular $\mathrm{I}_{2}$ and oxygen atoms (also created in the electric discharge section) that rapidly dissociate the $\mathrm{I}_{2}$ molecule. In this way $\mathrm{O}$ atoms play a positive role.

However, it was found that $\mathrm{O}$ atoms also play a significantly detrimental role through a rapid quenching of the desired excited $\mathrm{I}\left({ }^{2} \mathrm{P}_{1 / 2}\right)$ state, a reaction that was first hypothesized by the Emory University group. This particular rate proved to be challenging to measure, but was recently definitively determined, ${ }^{3,4}$ and found to be faster than originally thought. Our experiments showed that this reaction played the central role in previous failures to lase the ElectricOIL system. Once this problem was recognized, a mitigation method was devised to control the $\mathrm{O}$ atoms

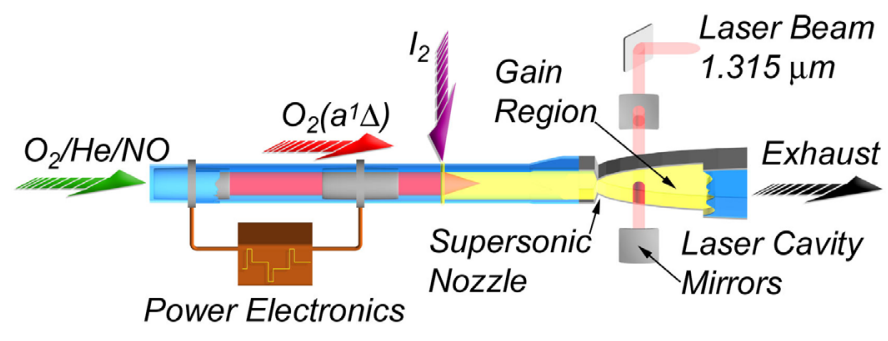

Figure 1. This schematic depicts the ElectricOIL system, a hybrid electric oxygen-iodine laser that transfers energy from $\mathrm{O}_{2}\left({ }^{1} \Delta\right)$ to atomic iodine. Helium lowers the gas flow temperature.

through the addition of small quantities of $\mathrm{NO}$ or $\mathrm{NO}_{2}$ to the gas mixture, which then facilitated successful gain and lasing demonstrations. ${ }^{1,2}$ Figure 2 shows a photograph of the system.

The evolution of this new hybrid laser technology continues. Future goals include enhancing the efficient production of the $\mathrm{O}_{2}$ (a) molecules, and determining the important kinetic processes in the discharge and post-discharge regions. 'Pulsersustainer' discharge concepts are being pursued by various groups in the United States that would increase the discharge ionization while also optimizing the power into the discharge plasma. In tandem, kinetic studies are being conducted aimed at making the laser system perform more efficiently as a whole.

Although this new technology is still in its infancy, some believe it can be enhanced to much higher power levels. This would make it a logical successor to the chemical oxygen-iodine laser (COIL) system being implemented in the Air Force's Airborne Laser (ABL) program. ElectricOIL's primary advantage is that it does not require the logistically problematic liquid singlet oxygen generators of the classic COIL system.

Since the initial ElectricOIL demonstrations, gain and power have been improved by an order of magnitude. Researchers from Russia, Japan, Czech Republic, and other groups in the United States have begun programs to study this fascinating, complex electrodynamic-kinetic laser system.

Continued on next page 


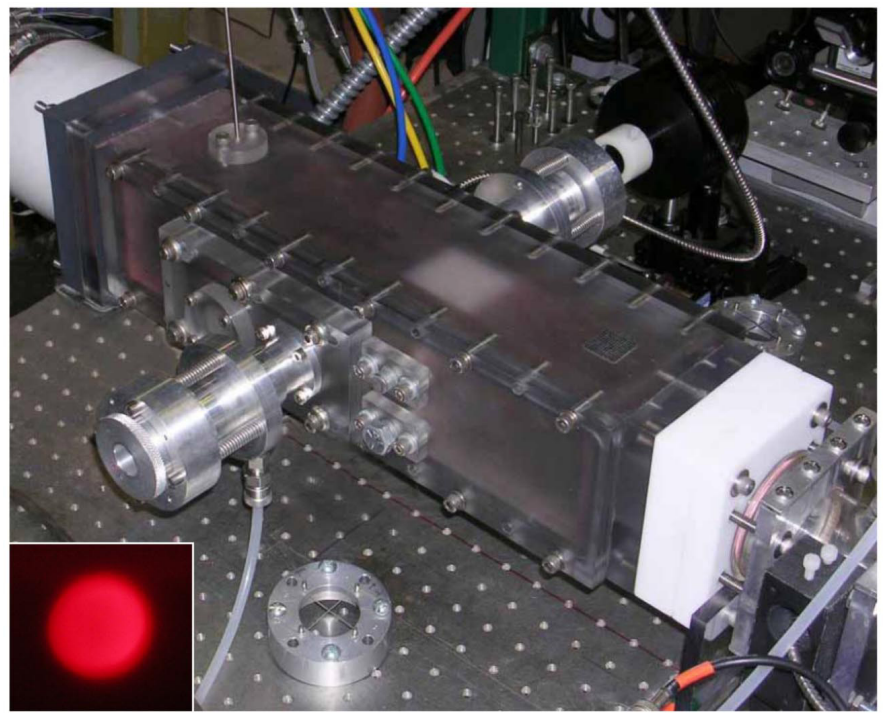

Figure 2. Shown here is the ElectricOIL supersonic laser cavity, with an inset of the laser beam as observed on a thermal image card.

The development of this new laser system was supported by Joint Technology Office funding through the Air Force Office of Scientific Research, and by Missile Defense Agency funding through the US Army Space and Missile Defense Command. Preliminary research was supported by the Air Force Research Lab, Directed Energy Directorate.

\section{Author Information}

\section{David Carroll}

CU Aerospace

Champaign, IL

\section{Wayne Solomon}

Aerospace Engineering

University of Illinois at Urbana-Champaign

Urbana, IL

\section{References}

1. D. L. Carroll, J. T. Verdeyen, D. M. King, J. W. Zimmerman, J. K. Laystrom, B. S. Woodard, N. Richardson, K. Kittell, and W. C. Solomon, Measurement of positive gain on the 1315nm transition of atomic iodine pumped by $\mathrm{O}_{2}\left({ }^{1} \Delta\right)$ produced in an electric discharge, App. Phys. Lett. 85 (8), pp. 1320-1322, 2004. doi:10.1063/1.1784519

2. D. L. Carroll, J. T. Verdeyen, D. M. King, J. W. Zimmerman, J. K. Laystrom, B. S. Woodard, G. F. Benavides, K. Kittell, D. S. Stafford, M. J. Kushner, and W. C. Solomon, Continuous-wave laser oscillation on the $1315 \mathrm{~nm}$ transition of atomic iodine pumped by $\mathrm{O}_{2}\left({ }^{1} \Delta\right)$ produced in an electric discharge, App. Phys. Lett. 86 (111104), pp. 1-3, 2005. doi:10.1063/1.1883317

3. V. N. Azyazov, I. Antonov, S. Ruffner, and M. Heaven, Quenching of $I\left({ }^{2} P_{1 / 2}\right)$ by $\mathrm{O}_{3}$ and $\mathrm{O}\left({ }^{3} P\right)$, SPIE 6101, p. 61011Y, 2006.

4. W. T. Rawlins, S. Lee, W. J. Kessler, L. G. Piper, and S. J. Davis, Advanced Diagnostics and Kinetics of Oxygen-Iodine Laser Systems, AIAA Paper, 2005-5299. 